\title{
Original
}

\section{Rehabilitation Approaches for Various Types of Dysphagia after Esophageal Cancer Surgery}

\author{
Yoshitaka $\mathrm{KII}^{1)}$ and Masazumi MizumA ${ }^{2)}$
}

\begin{abstract}
There are no reports reviewing the various types of dysphagia that occur after surgery for esophageal cancer and the rehabilitation approaches appropriate for each type. Herein, we classified various types of dysphagia after esophageal cancer surgery according to cause and examined the rehabilitation approaches for each type, as well as the outcomes. The study was performed on 31 patients who were tested postoperatively for swallowing function and underwent rehabilitation for dysphagia during their hospitalization. In patients with decreased swallowing ability due to recurrent laryngeal nerve injury associated with lymph node dissection, oral intake ability was improved by basic and feeding exercises. In patients with markedly reduced laryngeal elevation detected by videofluoroscopic examination of swallowing, feeding exercises were performed in addition to basic exercises that centered on stretching the perilaryngeal muscles. Improvements in laryngeal elevation were observed in some patients. In patients with dysphagia caused by postoperative gastrointestinal (GI) anastomotic stricture, the balloon dilatation method using an upper GI endoscope was applied at the anastomotic site. Finally, in patients with retention and reflux of GI tract contents in whom the reflux of contents into the pharynx caused aspiration, postures unlikely to cause reflux were maintained. Thus, dysphagia after esophageal cancer surgery may be due to not only swallowing dysfunction, but also impairments of the GI tract. When ingestion and swallowing abilities are assessed, simultaneous videofluoroscopic examination of swallowing and an upper GI series are important. In some cases, standard rehabilitation techniques alone may not be sufficient, requiring a multimodal approach for effective treatment.
\end{abstract}

Key words : esophageal cancer, dysphagia, rehabilitation, videofluoroscopic examination of swallowing, upper gastrointestinal series

\section{Introduction}

In Japan in 1981, cancer was ranked as the leading cause of death. Since then, the number of cancer cases has increased each year. There is a growing need for flexible rehabilita-

\footnotetext{
1) Department of Rehabilitation Medicine, Showa University Northern Yokohama Hospital, 35-1 Chigasaki-chuou, Tsuduki-ku, Yokohama 224-8503, Japan.

2) Department of Rehabilitation Medicine, Showa University School of Medicine.
} 
tion approaches not only to alleviate issues surrounding the cancer itself and the disabilities incurred during the course of treatment, but to also improve patients' activities of daily living ${ }^{1)}$. When the Ministry of Health, Labour and Welfare of Japan revised the system of remuneration for medical care services in 2010, it created a scheduled fee for the rehabilitation of cancer patients ${ }^{1)}$. Thus, there is an expectation of the involvement of rehabilitationrelated staff in the treatment of cancer patients.

One treatment for cancer, esophageal cancer surgery, which may require operations of the cervical, thoracic, and abdominal regions, is highly invasive, and extensive, and it imposes a major postoperative burden on patients. Advances in surgical procedures, surgical devices, anesthetic procedures, and postoperative pain control have resulted in greater safety of the technique. However, the incidence of postoperative complications following three-field lymph node dissection for esophageal cancer remains at approximately $60 \%{ }^{2}$. Although the long-term survival of patients is increasing as a result of progress in surgical therapy, chemotherapy, and radiotherapy ${ }^{3)}$, esophageal cancer remains an intractable malignancy. The frequency of postoperative complications is unchanged.

Respiratory complications after esophageal cancer surgery include respiratory failure, pulmonary edema, pneumonia, and atelectasis. Cardiovascular complications include arrhythmia and pulmonary infarction. Furthermore, other serious complications may occur, including anastomotic leak, recurrent laryngeal nerve palsy, pyothorax, chylothorax, wound infection, etc. Thus, this type of surgery is known to be associated with various, potentially serious, complications.

Of the disorders that arise as a result of complications of the surgery, dysphagia, respiratory dysfunction, and muscular weakness due to disuse are targeted by rehabilitation approaches. Esophageal cancer is regarded to result in disorders that require various rehabilitation approaches ${ }^{4)}$.

Dysphagia after esophageal cancer surgery has been reported to lead to pneumonia due to aspiration, as well as malnutrition resulting from decreased food intake ${ }^{5)}$, and although there are occasional reports on dysphagia after esophageal cancer surgery, its causes have not been investigated in detail and the underlying mechanisms remain unknown ${ }^{6}$. Furthermore, there are no reports reviewing the various types of dysphagia that may occur after esophageal cancer surgery and the rehabilitation approaches appropriate for each type. Classifiction of the different types of dysphagia arising as a complication of esophageal cancer surgery and the use of specific early rehabilitation approaches according to each type is expected to reduce the incidence of secondary complications, such as pneumonia caused by aspiration and malnutrition resulting from decreased food intake. A detailed investigation of different rehabilitation approaches may prove useful in improving the comprehensive medical care of patients with esophageal cancer.

In the present study, we identified the different types of dysphagia that occurred after esophageal cancer surgery and examined the rehabilitation approaches used for each. 


\section{Methods}

Of the 57 patients who underwent esophageal cancer surgery at Showa University Northern Yokohama Hospital between August 2009 and October 2011, 31 (29 men, two women) who had been tested postoperatively for swallowing function and had undergone various rehabilitation approaches for dysphagia during their hospitalization were included in the study. The reconstructive surgical procedures performed included retrosternal gastric tube reconstruction ( $n=17$ patients), antesternal gastric tube reconstruction $(n=13)$, and antesternal colon reconstruction $(n=1)$. All patients underwent three-field lymph node dissection, including cervical lymph node dissection (Table 1).

Based on past reports of postoperative complications ${ }^{7-10)}$, different types of dysphagia were identified in the present study on the basis of videofluoroscopic examination of swallowing and an upper gastrointestinal series as: (i) laryngeal movement, with or without pharyngeal residue; (ii) passage of test diets at an anastomotic site; (iii) retention; and (iv) reflux. Furthermore, the types of rehabilitation approaches for each type of disorder were evaluated.

In the present study, the "consultation period" was deemed to have started when we received a referral from the primary department and to end when videofluoroscopic examination of swallowing no longer revealed aspiration, regardless of inpatient or outpatient consultation. Thus, there were patients whose consultation period was completed because of the extremely low likelihood of aspiration, despite impaired swallowing function (e.g. slight pharyngeal residue after swallowing and delayed triggering of the swallowing reflex) being evident at the completion of the consultation.

\section{Results}

The different types of dysphagia, as determined by videofluoroscopic examination of swallowing and upper gastrointestinal series, and the rehabilitation approaches used are summarized in Table 2.

\section{Classification}

As noted in the Methods, videofluoroscopic examination of swallowing and an upper gastrointestinal series were used to identify the different types of dysphagia in the 31 patients in whom postoperative swallowing was evaluated.

a) Recurrent laryngeal nerve palsy

Patients with hoarseness in whom videofluoroscopic examination of swallowing revealed pharyngeal residue after swallowing were defined as having recurrent laryngeal nerve palsy. In the present study, recurrent laryngeal nerve palsy was identified in $22(71.0 \%)$ patients. The test findings for these patients revealed pyriform sinus residue after swallowing (Fig. 1) and, in 16 patients, the residue was observed on either the left side or on both sides.

b) Reduced laryngeal elevation 
Table 1. Patients and procedures

\begin{tabular}{|c|c|c|c|c|}
\hline Patient & Age & Sex & Reconstruction method & Lymph node dissection \\
\hline 1 & 72 & Male & A & \multirow{31}{*}{ 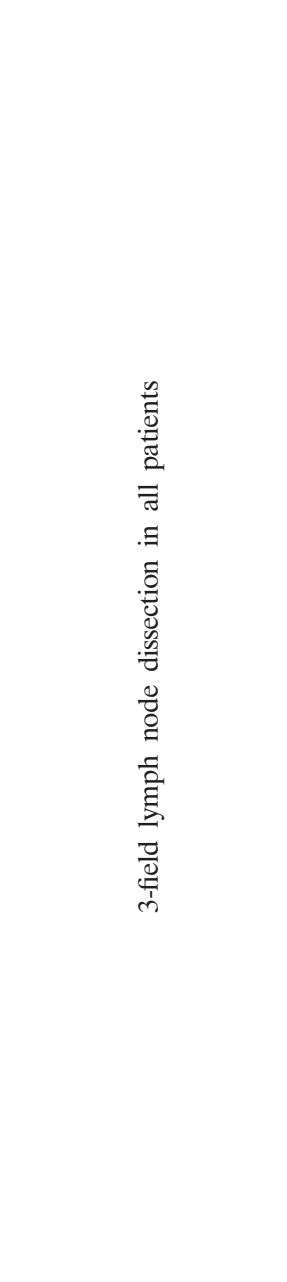 } \\
\hline 2 & 78 & Male & A & \\
\hline 3 & 66 & Male & A & \\
\hline 4 & 63 & Male & A & \\
\hline 5 & 65 & Male & A & \\
\hline 6 & 71 & Male & A & \\
\hline 7 & 68 & Male & A & \\
\hline 8 & 66 & Male & A & \\
\hline 9 & 76 & Male & $\mathrm{B}$ & \\
\hline 10 & 73 & Female & A & \\
\hline 11 & 73 & Male & A & \\
\hline 12 & 74 & Male & A & \\
\hline 13 & 58 & Male & $\mathrm{B}$ & \\
\hline 14 & 66 & Male & A & \\
\hline 15 & 61 & Female & $\mathrm{C}$ & \\
\hline 16 & 76 & Male & A & \\
\hline 17 & 77 & Male & A & \\
\hline 18 & 76 & Male & $\mathrm{B}$ & \\
\hline 19 & 68 & Male & $\mathrm{B}$ & \\
\hline 20 & 62 & Male & A & \\
\hline 21 & 78 & Male & B & \\
\hline 22 & 62 & Male & A & \\
\hline 23 & 60 & Male & A & \\
\hline 24 & 67 & Male & B & \\
\hline 25 & 63 & Male & B & \\
\hline 26 & 59 & Male & B & \\
\hline 27 & 55 & Male & B & \\
\hline 28 & 65 & Male & B & \\
\hline 29 & 76 & Male & B & \\
\hline 30 & 60 & Male & B & \\
\hline 31 & 52 & Male & B & \\
\hline
\end{tabular}

A : Retrosternal gastric tube reconstruction

$\mathrm{B}$ : Antesternal gastric tube reconstruction

$\mathrm{C}$ : Antesternal colon reconstruction

Patients in whom videofluoroscopic examination of swallowing revealed laryngeal movement corresponding to one vertebral body or less ${ }^{11)}$ were defined as having reduced laryngeal elevation. Three $(9.7 \%)$ patients in the present study were identified as having markedly reduced laryngeal elevation (Fig. 2).

c) Anastomotic stricture

Unlike preoperative esophageal stricture due to esophageal cancer, patients in whom an upper gastrointestinal series revealed passage of jelly, gruel, potage-like fluid, and/or liquid and confirmed retention at an enteric anastomotic site were defined as having anastomotic 
Table 2. Classification by dysphagia type and consultation period at our department

\begin{tabular}{|c|c|c|c|c|c|c|c|}
\hline Patient & Age & Sex & $\begin{array}{c}\text { Recurrent } \\
\text { laryngeal } \\
\text { nerve palsy }\end{array}$ & $\begin{array}{l}\text { Reduced } \\
\text { laryngeal } \\
\text { elevation }\end{array}$ & $\begin{array}{l}\text { Anastomotic } \\
\text { stricture }\end{array}$ & Reflux & $\begin{array}{l}\text { Period from start to end of consultation } \\
\text { at our department }\end{array}$ \\
\hline 1 & 72 & Male & & & & $\bigcirc$ & 4 weeks \\
\hline 2 & 78 & Male & & & & $\bigcirc$ & 3 weeks \\
\hline 3 & 66 & Male & 0 & & $\bigcirc$ & & 9 weeks \\
\hline 4 & 63 & Male & 0 & & & & 15 weeks \\
\hline 5 & 65 & Male & 0 & & $\bigcirc$ & & 25 weeks \\
\hline 6 & 71 & Male & $\bigcirc$ & & $\bigcirc$ & $\bigcirc$ & 24 weeks \\
\hline 7 & 68 & Male & 0 & & & & 11 weeks \\
\hline 8 & 66 & Male & 0 & & & & 15 weeks \\
\hline 9 & 76 & Male & 0 & & $\bigcirc$ & 0 & 36 weeks \\
\hline 10 & 73 & Female & 0 & & & & 6 weeks \\
\hline 11 & 73 & Male & 0 & & 0 & & 22 weeks \\
\hline 12 & 74 & Male & 0 & & & & 5 weeks \\
\hline 13 & 58 & Male & $\bigcirc$ & $\bigcirc$ & & & 17 weeks \\
\hline 14 & 66 & Male & & $\bigcirc$ & & & $\begin{array}{l}\text { (Laryngeal suspension and } \\
\text { cricopharyngeal myotomy) }\end{array}$ \\
\hline 15 & 61 & Female & 0 & & 0 & $\bigcirc$ & 15 weeks \\
\hline 16 & 76 & Male & & & $\bigcirc$ & & $\begin{array}{l}\text { (Only balloon dilatation method via } \\
\text { upper endoscopy) }\end{array}$ \\
\hline 17 & 77 & Male & $\bigcirc$ & $\bigcirc$ & & & 18 weeks \\
\hline 18 & 76 & Male & & & $\bigcirc$ & & $\begin{array}{l}\text { (Only balloon dilatation method via } \\
\text { upper endoscopy) }\end{array}$ \\
\hline 19 & 68 & Male & O & & & & 2 weeks \\
\hline 20 & 62 & Male & O & & & & 3 weeks \\
\hline 21 & 78 & Male & $\bigcirc$ & & $\bigcirc$ & & $\begin{array}{l}\text { (Incomplete termination due to } \\
\text { reoperation during dysphagia } \\
\text { rehabilitation approaches) }\end{array}$ \\
\hline 22 & 62 & Male & $\bigcirc$ & & & & 3 weeks \\
\hline 23 & 60 & Male & $\bigcirc$ & & & & 3 weeks \\
\hline 24 & 67 & Male & $\bigcirc$ & & & & 4 weeks \\
\hline 25 & 63 & Male & & & $\bigcirc$ & & $\begin{array}{l}\text { (Only balloon dilatation method via } \\
\text { upper endoscopy) }\end{array}$ \\
\hline 26 & 59 & Male & $\bigcirc$ & & & & 3 weeks \\
\hline 27 & 55 & Male & & & & $\bigcirc$ & 4 weeks \\
\hline 28 & 65 & Male & & & & & (Only meal patterns were checked) \\
\hline 29 & 76 & Male & O & & & & 4 weeks \\
\hline 30 & 60 & Male & $\bigcirc$ & & & & 3 weeks \\
\hline 31 & 52 & Male & & & & & (Only meal patterns were checked) \\
\hline \multicolumn{3}{|c|}{ Total } & 22 & 3 & 10 & 6 & \\
\hline
\end{tabular}

stricture. Postoperative anastomotic stricture (Fig. 3) was observed in 10 (32.3\%) patients in the present study.

d) Reflux of ingested food and / or digestive juice

Patients in whom an upper gastrointestinal series confirmed reflux were defined as having 


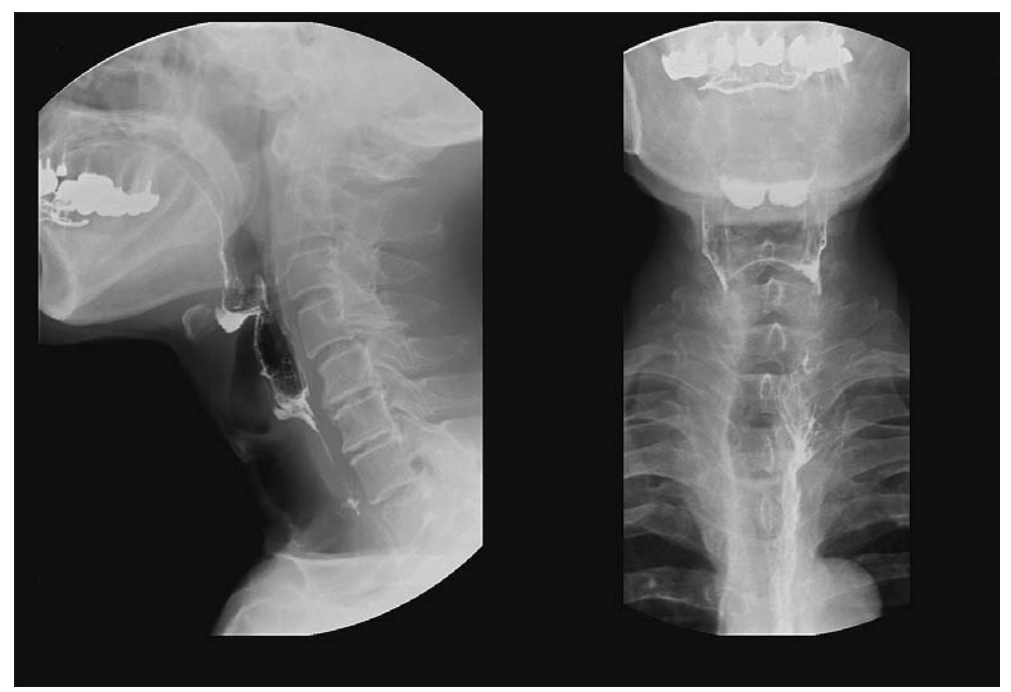

Fig. 1. Videofluoroscopic images from a patient with recurrent laryngeal nerve palsy, obtained while swallowing

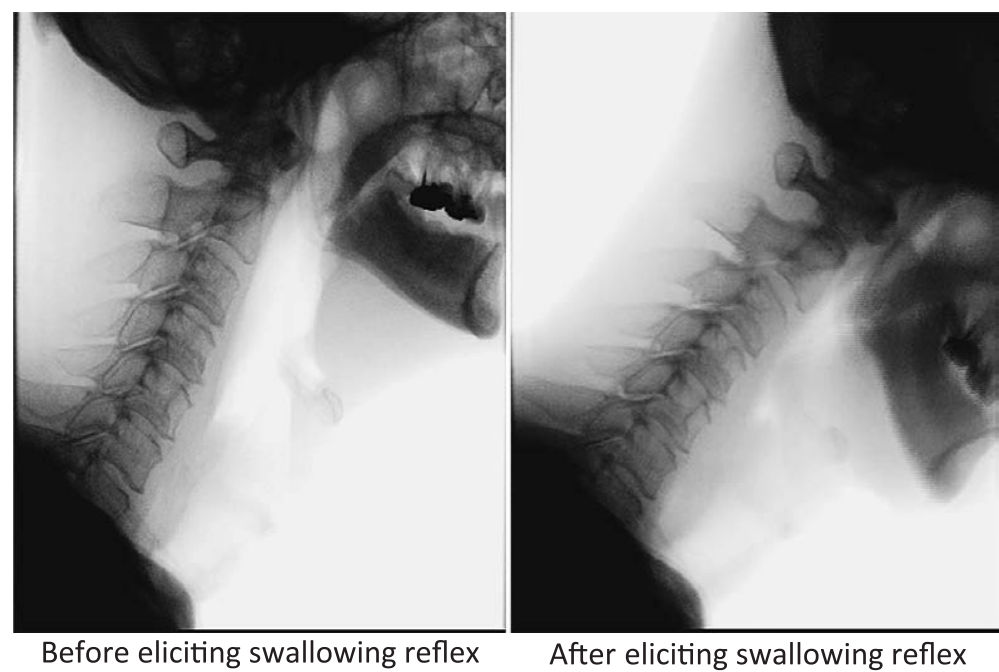

Fig. 2. Videofluoroscopic images from a patient with reduced laryngeal elevation, obtained while swallowing

reflux of ingested food and/or digestive juice. Six patients (19.4\%) in the present study were identified as having reflux (Fig. 4).

Rehabilitation approaches for each type of dysphagia

a) Rehabilitation for recurrent laryngeal nerve palsy

Various rehabilitation approaches were used for patients who had recurrent laryngeal nerve palsy. All 22 patients performed the tongue holding maneuver ${ }^{12)}$, which helps 


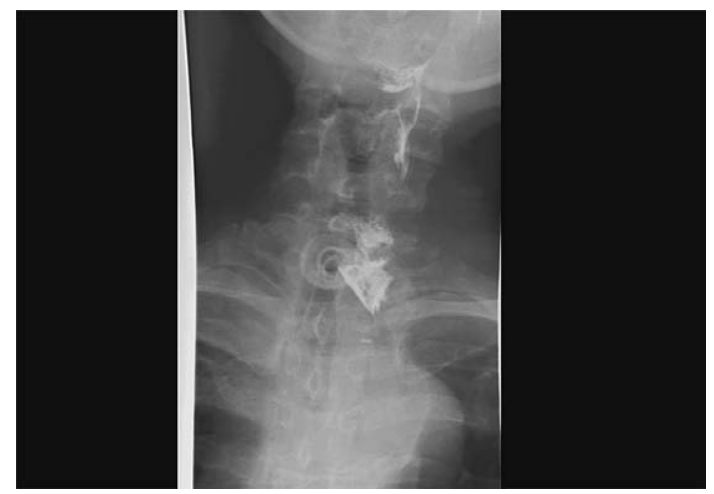

Fig. 3. Videofluoroscopic image from a patient with anastomotic stricture, obtained while swallowing

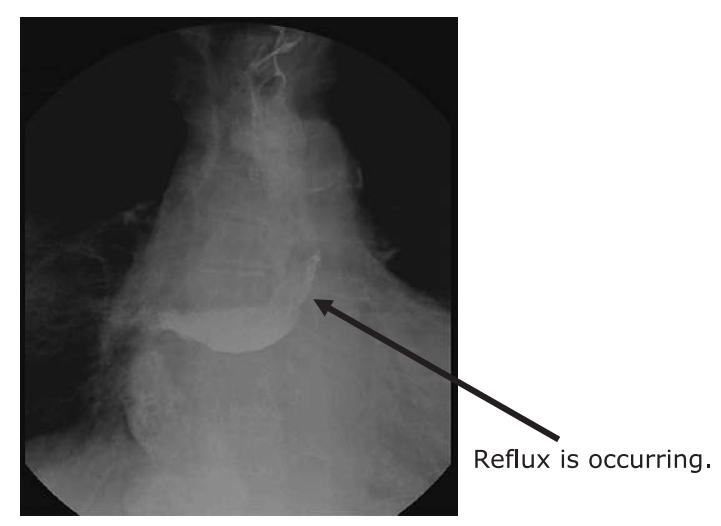

Fig. 4. Videofluoroscopic image from a patient with reflux, obtained while swallowing

improve pharyngeal constriction and reduces pharyngeal residue, as well as the head-raising exercise $^{13)}$, which improves dilatation of the esophageal orifice. Nine patients further performed the head rotation procedure ${ }^{14)}$, in which they rotated their neck to open one side of the pharynx and swallowed to remove the pharyngeal residue. In addition, postural and dietary modifications were applied to all 22 patients.

These approaches improved swallowing function. Over a consultation period of $2 \sim 36$ weeks, videofluoroscopic examination of swallowing no longer revealed aspiration and thus treatment in Department of Rehabilitation Medicine was deemed to have been completed. However, among these patients, there were some in whom swallowing function was impaired not only because of recurrent laryngeal nerve palsy, but also anastomotic stricture and reflux. In patients in whom aspiration could be attributed to recurrent laryngeal nerve palsy, videofluoroscopic examination of swallowing ceased to reveal aspiration after $2 \sim 24$ weeks. Patients with a consultation period of 25 weeks had swallowing difficulties due to anasto- 


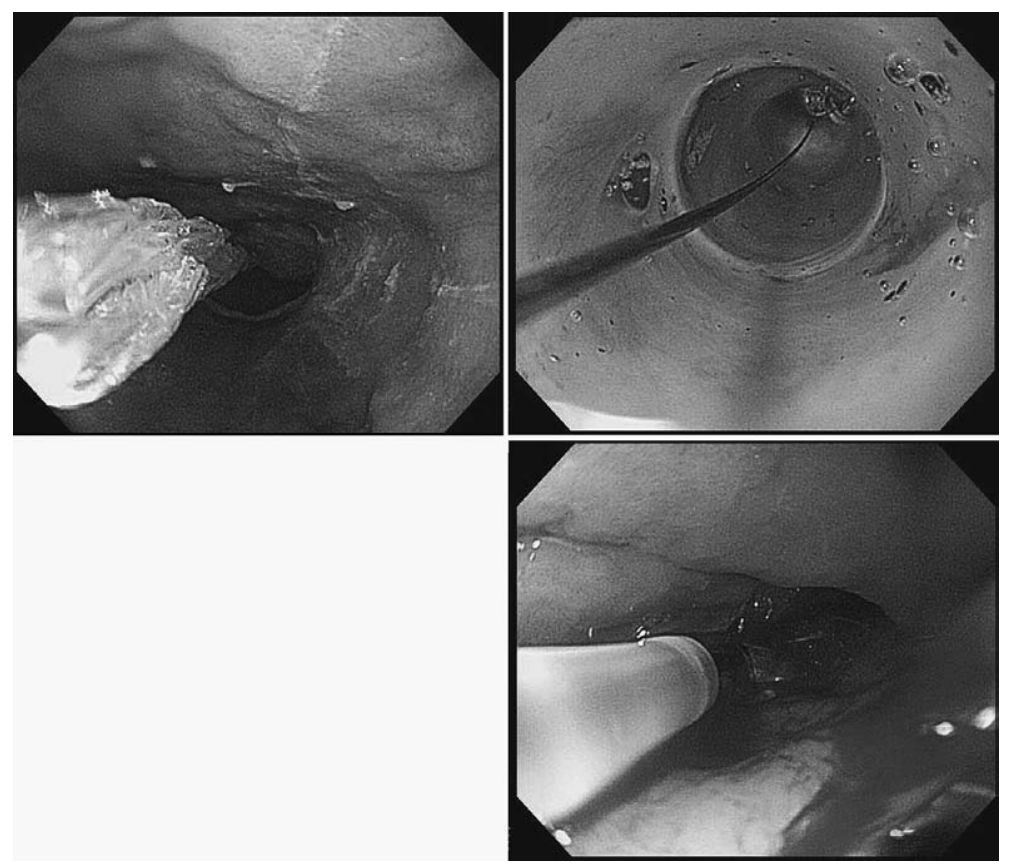

Fig. 5. Endoscopic balloon dilatation method

motic stricture and continued treatment with their primary doctor at the outpatient unit and Department of Rehabilitation Medicine. Those patients who required 36 weeks consultation had aspiration due to reflux and so their consultation period was extended.

b) Rehabilitation for reduced laryngeal elevation

All three patients in whom reduced laryngeal elevation was identified performed basic exercises ${ }^{15)}$ centered on stretching the perilaryngeal muscles, as well as feeding exercises ${ }^{16)}$, in which they ate foods that were unlikely to cause aspiration. Although an improvement in laryngeal elevation was observed in two patients in $17 \sim 18$ weeks, there was no change in the condition of the third patient.

c) Rehabilitation for anastomotic stricture

In patients in whom anastomotic stricture was identified, an endoscopist performed endoscopic balloon dilatation (Fig. 5).

d) Rehabilitation for reflux of ingested food and digestive juice

To improve reflux of ingested food and digestive juice, all six patients in this group were advised to keep their head elevated by $15^{\circ} \sim 20^{\circ}$ while lying down. Moreover, the daily food intake was divided into five to six smaller meals in two patients and proton pump inhibitors were administered for three patients undergoing gastric tube reconstruction. Although there were various underlying causes of the dysphagia in some patients, relief was observed in 3 36 weeks, after which the consultation period in Department of Rehabilitation Medicine was finished. 


\section{Discussion}

\section{Reconstructive surgical methods and dysphagia}

When considering surgical procedures for esophageal cancer at Showa University Northern Yokohama Hospital, antesternal gastric tube reconstruction is selected for patients undergoing preoperative radiotherapy, the elderly (those $\geq 80$ years), patients with concurrent diabetes mellitus or renal dysfunction, and some patients undergoing reoperation due to anastomotic leak. Thus, of the patients undergoing antesternal gastric tube reconstruction, none or only a very few may have had impaired swallowing function due to a poor systemic condition immediately prior to surgery. With regard to postoperative dysphagia, regardless of the reconstructive surgical method used, patients referred to the Department of Rehabilitation may have been deemed able to start oral intake by their primary doctors.

\section{Recurrent laryngeal nerve palsy}

During esophageal cancer surgery, lymph node dissection in the cervical region is performed along the right and left recurrent laryngeal nerves located in the paraesophageal area. This area is known to be the most common site of lymph node metastasis in esophageal cancer and is the most important site of dissection. However, the operation itself may cause recurrent laryngeal nerve palsy ${ }^{4)}$. The recurrent laryngeal nerves are regarded as motor nerves distributed to muscles associated with airway closure and muscles at the pharyngoesophageal junction ${ }^{17)}$. Thus, injury to the recurrent laryngeal nerves may result in impaired swallowing, characterized by vocal cord dysraphism, obstruction by the cricopharyngeus muscle, and pyriform sinus residue after swallowing. Moreover, the left recurrent laryngeal nerve, which runs lengthwise in the mediastinum, is likely to be affected by the dissection process ${ }^{18)}$. This may explain why recurrent laryngeal nerve palsy is common on the left side. Of the patients in the present study, 16 had residue on the left or both sides, which may reflect an effect of the surgical procedures on the left recurrent laryngeal nerve.

With regard to rehabilitation approaches for dysphagia, the balloon dilatation method ${ }^{19)}$, which is often used to relieve dysphagia due to bulbar palsy, was not used on any of the patients in the present study. Swallowing function is likely to have improved in our patients mainly due to the basic subjective exercises that they performed. Such improvements may reflect milder obstruction by the cricopharyngeus muscle and pyriform sinus residue after swallowing compared with bulbar palsy. These disorders do not involve the swallowing center. Even though lymph node dissection damages the recurrent laryngeal nerves, the damage is partial and the disorder is relatively mild. Thus, improvements in swallowing can be expected by asking patients to perform subjective exercises, rather than painful exercises. In the present series, swallowing had improved sufficiently so as not to cause aspiration after 2 24 weeks, which is consistent with results reported previously ${ }^{20)}$. Swallowing may be expected to improve gradually using standard rehabilitation techniques, including basic 
exercises. The variability in the time until no aspiration was detected by videofluoroscopic examination suggests that nerve damage was caused by the surgical procedures. If recurrent laryngeal nerves are severely damaged, recovery can be expected to take relatively longer.

\section{Reduced laryngeal elevation}

It has been reported that adhesion caused by cervical lymph node dissection reduces laryngeal elevation ${ }^{8)}$. Another study has reported that anterior cervical myotomy during surgery on the cervical region possibly reduces laryngeal elevation ${ }^{21)}$. Of the anterior cervical muscles, infrahyoid muscles may be affected during surgical procedures on the cervical region and, of the infrahyoid muscles, the thyrohyoid muscle is involved in laryngeal elevation ${ }^{22)}$. Despite this, laryngeal elevation was not observed in any of the patients in the present series in whom ablation of the thyrohyoid muscle was confirmed. Ablation of infrahyoid muscles other than the thyrohyoid muscle (i.e. the sternohyoid, sternothyroid, and omohyoid muscles) reportedly facilitates laryngeal elevation during swallowing ${ }^{23)}$. However, assessment of laryngeal elevation is difficult if only the effect of cervical dissection is taken into consideration ${ }^{24)}$. Anterior cervical myotomy as a factor reducing laryngeal elevation is contentious and is an issue that warrants further investigation in future studies. If a reduction in laryngeal elevation is due to postoperative adhesions, it may be improved by performing basic exercises centered on stretching the perilaryngeal muscles and feeding exercises ${ }^{15,25)}$. Indeed, laryngeal elevation was improved in two patients in the present series using these approaches. However, no improvement was observed in a third patient with laryngeal elevation. A similar lack of improvement has been reported previously ${ }^{7)}$ and, in such cases, laryngeal suspension or ablation of infrahyoid muscles other than the thyrohyoid muscle may be necessary ${ }^{23)}$. It may also be necessary to remain flexible in terms of the rehabilitation approach chosen, for example not simply using rehabilitation techniques, but also considering surgical treatment.

\section{Anastomotic stricture}

The cause of anastomotic stricture is an organic stricture of an enteric anastomotic site following surgery. There are two procedures for dilatation therapy for esophageal strictures: (i) endoscopic balloon dilatation, in which the tissues of a stricture site are stretched temporarily or torn in anticipation of long-term effects of treatment; and (ii) placement of an esophageal stent, in which semipermanent dilatation is expected. If the anastomotic site after upper gastrointestinal tract surgery is considered to represent a benign condition, stent placement for stricture is generally avoided because of the high risk of hemorrhage or perforation, complications that are not warranted by a benign disorder. Thus, endoscopic balloon dilatation is used for the treatment of anastomotic stricture ${ }^{26)}$.

It has been reported that $45 \%$ of patients undergo endoscopic balloon dilatation for anastomotic stricture ${ }^{27)}$. Although the frequency of the procedure in the present study was 
lower, anastomotic stricture after esophageal cancer surgery has been suggested to occur at a certain predictable rate. If a patient has anastomotic stricture, it can be assumed that not only will there be difficulties with the passage of food, but that aspiration may also occur due to the reflux of ingested food. Thus, anastomotic stricture after esophageal cancer surgery needs to be treated as a type of dysphagia.

At Showa University Northern Yokohama Hospital, anastomotic strictures are subject to detailed endoscopic examination, with the findings used as a reference to determine when oral intake can be started. However, there are patients who develop obstructions after they start the oral intake of food. In the present study, obstruction was noted in two patients. Although some other patients without complications were initially capable taking food orally, they subsequently developed anastomotic strictures. Thus, with regard to the treatment of dysphagia, if a patient complains of aphagia, both the pharynx and esophagus should be examined. The diagnosis of an anastomotic stricture requires not only observation of swallowing movements in the oral cavity or pharynx, but also determination of passage rates using an upper gastrointestinal series. If anastomotic stricture is diagnosed, standard rehabilitation techniques will be insufficient and endoscopic treatment may well be necessary.

\section{Reflux}

After esophageal cancer surgery, because of the loss of mechanisms to prevent reflux, gastrointestinal hypomotility etc., there is a higher frequency of the retention of digestive juices and ingested food in the reconstructed organs, which can lead to reflux. The incidence of reflux after gastric tube reconstruction has been reported to be in the range $60 \% \sim 80 \%{ }^{10}$. It is not uncommon for refluxed material to reach the pharynx and larynx, and so aspiration pneumonia is common ${ }^{28)}$. Reflux was observed in some of the patients in the present series. We advocate that reflux be included in the differential diagnosis for the treatment of dysphagia.

When an upper gastrointestinal series reveals retention or reflux of contrast medium in the reconstructed organs, it can be assumed that dysphagia may develop. However, there are some signs of dysphagia that cannot be detected without actually starting oral intake, such as vomiting and night-time choking, as well as subsequent fever and pneumonia. Thus, observation of patients is important.

With regard to the rehabilitation approaches for dysphagia, reflux cannot be treated with standard rehabilitation techniques. However, dietary and postural modifications, as well as drug therapy, may be effective in patients undergoing gastric tube reconstruction, such as dividing daily food intake into more than three meals, not lying down immediately after a meal, maintaining head elevation while lying down, including at bedtime, and taking oral proton pump inhibitors ${ }^{28)}$. In the present study, we divided daily food intake into five to six relatively smaller meals for those patients with reflux. In addition, when patients were recumbent during their hospitalization, we ensured that their head was elevated by $20^{\circ}$. 
Finally, patients were started on oral lansoprazole $15 \mathrm{mg}$ or omeprazole $10 \mathrm{mg}$. In all patients, reflux and choking were reduced, and fever and pneumonia were relieved. It should be noted that there is one study reporting that the administration of proton pump inhibitors does not have any effect on the total number of reflux episodes, even though these drugs do prevent acid from being released into refluxed contents ${ }^{29)}$. No consensus has been reached on the effects of proton pump inhibitors on functional dyspepsia. Although the use of proton pump inhibitors in the treatment of reflux and choking is contentious, postural modifications are an important first step in managing affected patients. Because the success of these rehabilitation approaches relies on both nursing care and the cooperation of the patients and their families, a team approach that includes the patient is essential.

\section{Necessary approaches for doctors managing dysphagia}

Dysphagia after esophageal cancer surgery is caused by hypofunction and organic alterations. When swallowing ability is assessed after esophageal cancer surgery, it is important to assess the contribution of different underlying causes to the dysphagia. To this end, both video endoscopic evaluation of swallowing, which is frequently used in clinical practice and has been reported to be useful ${ }^{30)}$, and simultaneous videofluoroscopic examination of swallowing and an upper gastrointestinal series should be performed.

If doctors managing dysphagia undertake both functional and organic assessments, they can suggest appropriate rehabilitation techniques, as well as endoscopic treatment, drug therapy, and dietary and postural modifications as needed. The results of functional and organic assessments will provide valuable information, enabling doctors to choose a greater range of therapeutic strategies to manage these patients appropriately. Treatment of dysphagia associated with esophageal cancer requires such a flexible approach. The results of the present study highlight the need to select the most appropriate approach in the treatment of the different types of dysphagia that may arise after esophageal cancer surgery.

\section{References}

1) Tsuji T: Cancer rehabilitation: current developments and the future. Jpn J Rehabil Med 47:296-303 (2010) (in Japanese)

2) Fang W, Kato H, Tachimori $Y$, Igaki H, Sato $H$ and Daiko $H$ : Analysis of pulmonary complications after three-field lymph node dissection for esophageal cancer. Ann Thorac Surg 76:903-908 (2003)

3) Ide H, Udagawa H, Ozawa S, Tachimori H, Saito T, Shinoda M, Tanaka O, Matsubara H, Yamana H and Barron JP: Overall survival curves of patients treated by esophagectomy (1988-1997). In: Comprehensive Registry of Esophageal Cancer in Japan, 3rd ed, The Japan Esophageal Society (Eds), Chiba, Japan, pp 158 (2002)

4) Seto Y, Fukuda S and Yamaguchi T: Standard operation for esophageal cancer 1. J Clin Rehabil 16:312-315 (2007) (in Japanese)

5) Atkins BZ, Fortes DL and Watkins KT: Analysis of respiratory complications after minimally invasive esophagectomy : preliminary observation of persistent aspiration risk. Dysphagia 22 : 49-54 (2007)

6) Shiozaki H, Yano M, Tamura S, Yasuda T, Fujiwara Y and Monden M: Swallowing function recovery operation 
for esophageal cancer. Operation 55:1087-1092 (2001) (in Japanese)

7) Namba T, Ito I, Tadenuma $T$, Sakai $Y$ and Maniwa S: A case report of the patient with bilateral vocal cord paralysis and dysphagia after esophagectomy. Bull Shimane Univ Fac Med 29: 1-7 (2006) (in Japanese)

8) Hou H, Imamoto H, Shiozaki H, Saito T, Nishino H, Miki H and Kanno M: Perioperative dysphagia rehabilitation for esophageal cancer. Otol Fukuoka 54:S236-S237 (2008) (in Japanese)

9) Kawano T: Dysphagia after esophageal cancer operation. Otol Fukuoka 47 : 119-121 (2001) (in Japanese)

10) Aly A and Jamieson GG: Reflux after oesopagectomy. Br J Surg 91 : 137-141 (2004)

11) Ueda K, Okada S, Kitazumi E, Koyama T, Takahashi K, Takehara I, Tanimoto K, Tsubahara A, Baba M, Horiguchi T, Yamamoto H and Fujisshima I : Videofluoroscopic examination of swallowing. Jpn J Dysphagia Rehabil 15: 76-95 (2011) (in Japanese)

12) Fujiu $\mathrm{M}$ and Logemann JA : Effect of a tongue-holding maneuver on posterior pharyngeal wall movement during deglutition. Am J Speech Lang Pathol 5 :23-30 (1996)

13) Shaker R, Easterling C, Kern M, Nitschke T, Massey B, Daniels S, Grande B, Kazandjian M and Dikeman K: Rehabilitation of swallowing by exercise in tube-fed patients with pharyngeal dysphagia secondary to abnormal UES opening. Gastroenterology 122 : 1314-1321 (2002)

14) Fujishima I : Feeding exercise. In: Rehabilitation for Swallowing Disorders associated with Stroke, 2nd ed, Ishiyaku Publishers, Tokyo, pp 116-120 (1998)

15) Okada S: Basic exercise for adult dysphagia. In: Dysphagia Rehabilitation, 2nd ed, Kamakura Y, Kumakura I, Fujishima I, Yamada Y, Baba T and Hironaka S (Eds), Ishiyaku Publishers, Tokyo, pp 180-184 (2007)

16) Shimizu M: Feeding exercise. In: Rehabilitation for Dysphagia, Ishiyaku Publishers, Ogura O, Shimizu M, Tanimoto K, Honda T and Mizojiri G (Eds), Tokyo, pp 235-254 (1998)

17) Crary MA and Groher ME: Neurologic control of swallowig. In : Introduction to Adult Swallowing Disorders, Butterworth-Heinemann, St. Louis, pp 31-36 (2003)

18) Seto S, Fukuda S and Yamaguchi T: Standard operation for esophageal cancer 2. J Clin Rehabil 16:404-407 (2007) (in Japanese)

19) Sumiya N : Rehbilitation of pharyngeal dysphagia. Intermittent air stretching method with balloon catheter. Gen Rehabil 20 : 513-516 (1992) (in Japanese)

20) Pierie JP, Goedegebuure S, Schuerman FA and Leguit P: Relation between functional dysphagia and vocal cord palsy after transhiatal oesophagectomy. Eur J Surg 166:207-209 (2000)

21) Tanuma A : Dysphagia rehabilitation for cancer patients. MB Med Rehabil 111 : 17-20 (2009)

22) Nakamura R and Saito H: Muscles of neck. In: Fundamental Kinesiology, 4th ed, Ishiyaku Publishers, Tokyo, pp 241-243 (1995)

23) Sakurai K : Surgery treatment for dysphagia. Mod Physician 26:82-83 (2006) (in Japanese)

24) Adachi K, Umezaki T, Kiyohara H, Shiratsuchi H, Nakashima $\mathrm{T}$ and Komune $\mathrm{S}$ : The effect of a neck dissection on the swallowing function. Otol Fukuoka 53:235-241 (2007) (in Japanese)

25) Sugiura J, Fujimoto Y, Ando A, Shimoda I and Nakashima T: Effects of manual muscle strengthening exercise on swallowing function in post-operative head-neck cancer patients with dysphagia. Jpn J Dysphagia Rehabil 12: 69-74 (2008) (in Japanese)

26) Aiko S and Kumano I: Expansion treatment for esophageal stenosis. J Adult Dis 40 : 960-966 (2010) (in Japanese)

27) Aiko S, Yoshizumi Y, Ogawa H, Ishizuka T, Horio T, Kanai N, Nakayama T and Maehara T: Surgical attempts to avoid anastomotic leaks and reduce reflux esophagitis following esophagectomy for cancer. Esophagus 5: 141-148 (2008)

28) Nishimura K, Fujita H, Tanaka T, Tanaka Y, Matono S, Murata K, Umeno H and Shirouzu K: Correlation between new classification system for reflex laryngopharyngitis and Los Angeles classification system for reflex esophagitis. J Jpn Bronchoesophagol Soc 60:268-275 (2009) (in Japanese)

29) Yano F, Kasiwagi H, Omura N, Tsuboi K, Tanishima Y, Matsumoto A, Hoshino M, Yamamoto S, Akimoto S and Yanaga K: Influence of proton pump inhibitors on gastroesophageal reflux in patients with nonerosive 
reflux disease (NERD). Proc Jpn Forum Stud Stomach 41:28 (2009) (in Japanese)

30) Hisa Y, Omae Y, Tayama J, Baba H, Hyodo M, Horiguchi T, Hirose H and Nakayama T: Video endoscopic evaluation of swallowing. In : Dysphagia Medical Treatment Guidelines, The Oto-Rhino-Laryngological Society of Japan, Inc. (Eds), Kanehara, Tokyo, pp 15-19 (2008)

[Received March 13, 2012 : Accepted April 20, 2012] 